\title{
Massive Star Asteroseismology in Action
}

\author{
Conny Aerts $\mathbf{s}^{1,2}$ \\ ${ }^{1}$ Institute of Astronomy, KU Leuven, Celestijnenlaan 200 D, 3001 Leuven, Belgium \\ email: Conny. Aerts@ster . kuleuven. be \\ ${ }^{2}$ Department of Astrophysics/IMAPP, Radboud University Nijmegen, 6500 GL Nijmegen, \\ the Netherlands
}

\begin{abstract}
After highlighting the principle and power of asteroseismology for stellar physics, we briefly emphasize some recent progress in this research for various types of stars. We give an overview of high-precision high duty-cycle space photometry of OB-type stars. Further, we update the overview of seismic estimates of stellar parameters of OB dwarfs, with specific emphasis on convective core overshoot. We discuss connections between pulsational, rotational, and magnetic variability of massive stars and end with future prospects for asteroseismology of evolved OB stars.
\end{abstract}

Keywords. asteroseismology, stars: oscillations (including pulsations), stars: interiors, stars: evolution, stars: rotation, methods: data analysis, methods: statistical, magnetic fields, waves, turbulence

\section{Progress in Asteroseismology}

Asteroseismology is a fairly new and powerful way of studying stellar physics, including important excursions to exoplanetary science and the study of galactic structure and evolution. Even in the context of stellar interiors alone, there are many aspects to this research field (e.g., Aerts et al. 2010a, for an extensive monograph). A single snapshot picture capturing the basic idea of seismic modelling is provided in Fig. 1, where $X, Z, M, \tau$ are the initial hydrogen fraction and metallicity, the mass, and the age, respectively. In this sketch, we assumed the model computations to be based on the simplistic (but practical!) one-dimensional time-independent mixing-length theory of convection described by the two free parameters $\alpha_{\mathrm{MLT}}$ and $\alpha_{\mathrm{ov}}$, which are the mixing length and convective overshoot parameters, both expressed in units of the local pressure scale height. For core-hydrogen burning massive stars, the choice of $\alpha_{\mathrm{MLT}}$ is of limited importance as long as it is within reasonable limits (e.g., between 1.5 and 2.0), while the poorly known amount of convective overshooting expressed by $\alpha_{\text {ov }}$ plays a pivotal role in their structure, in all of the evolutionary phases. This $\alpha_{\mathrm{ov}}$ is thus a key parameter to estimate and asteroseismology is a relatively new way to do so, as we will discuss further on.

With the high-precision space photometry from the CoRoT and Kepler missions at hand for thousands of FGK-type stars, priority was given to the determination of basic seismic observables and stellar parameters of such stars. The most popular observables are the so-called large and small frequency separations, which are the inverse of twice the sound travel time between the centre and the surface of the star and a measure for the sound-speed gradient in the stellar interior, respectively. The frequency of maximum power is also an important basic observable. Stochastically-excited modes are easy to identify as they form specific ridges in so-called échelle diagrams, in which the detected frequencies are reduced modulo the large frequency separation. Moreover, the seismic diagnostics are easily interpretable in terms of the fundamental parameters of the stars by means of scaling relations (e.g., Kallinger et al. 2010; Huber et al. 2011; Hekker et al. 


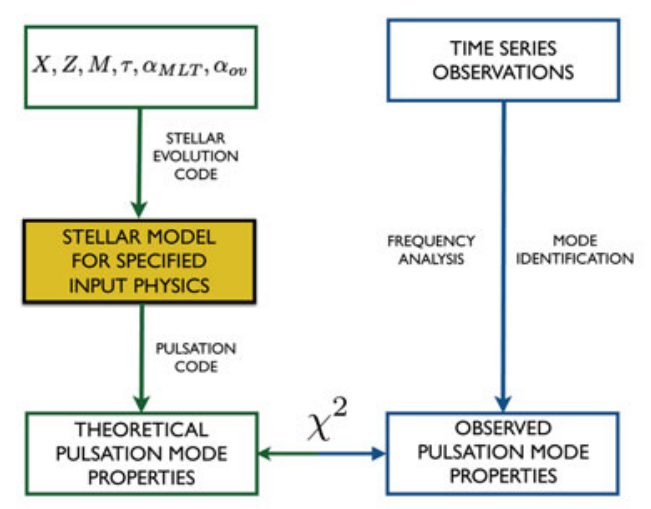

Figure 1. The principle of asteroseismic modelling in a snapshot, where the dependency on the chosen input physics of the equilibrium models is highlighted in shaded grey. Figure courtesy of Dr. Katrijn Clémer.

2011). The scheme represented in Fig. 1 hence delivers high-precision stellar parameters that are typically an order of magnitude more precise than those derived from classical ground-based snapshot spectroscopy or multicolour photometry (e.g., Chaplin et al. 2014), even taking into account uncertainties in the input physics. The seismic parameters derived from scaling relations are particularly welcomed for host stars of exoplanets (e.g., Gilliland et al. 2011; Huber et al. 2013; Chaplin et al. 2013; Van Eylen et al. 2014; Lebreton \& Goupil 2014) and for galactic clusters and population studies (e.g., Corsaro et al. 2012; Miglio et al. 2013; Stello et al. 2013; Casagrande et al. 2014).

The discovery of dipole mixed modes in low-mass evolved stars (Beck et al. 2011) allowed to go beyond the use of simple scaling relations and led to the derivation of their nuclear burning phase and hence evolutionary stage (Bedding et al. 2011). Such information is not accessible from classical data because core-helium burning and hydrogen-shell burning red giants have the same surface properties. Moreover, after two years of monitoring with the Kepler satellite, the detected rotational splitting of dipole mixed modes led to the derivation of their interior rotational properties, with core rotation typically only a factor 5 to 20 faster than envelope rotation (Beck et al. 2012; Mosser et al. 2012; Deheuvels et al. 2014), pointing to major shortcomings in our understanding of the angular momentum distribution inside stars (Eggenberger, these proceedings).

Many remarkable seismic studies of other types of stars are not mentioned here, due to space constraints and for that reason, we also limit the rest of the paper to massive stars. Their variability is far more diverse than that of low-mass stars due to phenomena like fast rotation, mass loss, close binarity, magnetism, etc. Hence, the case of massive OB star asteroseismology is challenging. A specific challenge is connected with the nature of the oscillations, i.e., the majority of the detected oscillation modes in massive stars are self-driven by a heat mechanism, which operates along with a yet unknown mode selection mechanism (e.g., Aerts et al. 2010a). This creates sparse low-order mode frequency spectra roughly in the range of 30 to $200 \mu \mathrm{Hz}$ and/or dense high-order gravitymode spectra at low frequencies, typically below $20 \mu \mathrm{Hz}$. Moreover, the rotation of most massive stars is such that their rotationally split pulsation mode multiplets get merged in frequency spectra, preventing pattern recognition as for high-order pressure modes in 
low-mass stars from échelle diagrams. In addition to these inherent difficulties based on the physics of the pulsations of such stars, OB-type targets have been avoided as much as possible in the fields of the CoRoT and Kepler exoplanet programmes, to maximise the efficiency of the search for transiting planets. Despite this limitation, progress in the asteroseismology of OB stars the past decade is large and is our point of focus in the remainder of this paper.

\section{Asteroseismic Data of OB Stars in the Space Age}

OB stars have been part of the space photometry revolution since a decade now, with observations from the MOST, CoRoT, and Kepler satellites. We review the observational studies based on space photometry and refer to the mentioned papers for the exquisite light curves and the accompanying Fourier spectra of the oscillation frequencies.

\subsection{Core-Hydrogen Burning Massive Stars}

MOST was a pioneering mission for massive stars. Although it could only monitor stars for typically up to 6 weeks, resulting in limited frequency precision, and with relatively low brightness precision (typically $0.1 \mathrm{mmag}$ ), it allowed to detect at least twice as many modes than known from the ground for selected $\beta$ Cep stars (Aerts et al. 2006b; Handler et al. 2009), to detect pulsations in Be stars (Saio et al. 2007), and to discover new Slowly Pulsating B stars (Aerts et al. 2006a; Cameron et al. 2008; Gruber et al. 2012).

The asteroseismology programme of the CoRoT mission consisted of long runs of five months (frequency precision $\sim 0.1 \mu \mathrm{Hz}$ ) as well as short runs of a few weeks, dedicated to bright stars (visual magnitude between 6 and 9) resulting in amplitude precision of roughly $10 \mu \mathrm{mag}$. This programme included detailed studies of carefully selected B stars and showed that the majority of them is pulsating in numerous modes, some of which with clear period spacings (Degroote et al. 2010a; Pápics et al. 2012) while others have less structured dense frequency spectra (Degroote et al. 2009b; Thoul et al. 2013). Some of the B stars turned out to be rotational variables without pulsations (Degroote et al. 2011; Pápics et al. 2011). One short run of CoRoT was dedicated to the monitoring of $\mathrm{O}$ stars during three weeks. These data showed diversity in the variability patterns with one $\beta$ Cep-type pulsator (Briquet et al. 2011), one primary of a long-period binary with stochastic modes (Degroote et al. 2010b), three stars with power excess at low frequency of unknown origin (Blomme et al. 2011) and one complex O-type binary, also known as Plaskett's star (Mahy et al. 2011). In addition, the CoRoT asteroseismology programme contained a number of Be stars (Neiner et al. 2009; Gutiérrez-Soto et al. 2009; Diago et al. 2009; Huat et al. 2009; Desmet et al. 2010; Emilio et al. 2010; Neiner et al. 2012a). These Be stars also revealed quite diverse variability patterns, including an outburst measured in real time for the B0.5IVe star HD 49330 due to the beating of non-radial pulsation modes, stochastically excited gravito-inertial modes, rotational modulation, and accretion phenomena in a close binary.

Numerous B dwarfs were also found in the CoRoT exoplanet programme (Degroote et al. 2009a), after variability classification by means of multivariate Gaussian mixtures (Debosscher et al. 2009; Sarro et al. 2009) supported by ground-based follow-up spectroscopy (Sarro et al. 2013). Similar studies have been done for the Kepler field (Debosscher et al. 2011), along with manual searches for B pulsators (Balona et al. 2011), resulting in the detection of variability with similar precision in amplitude than with CoRoT but with a frequency resolution $\sim 0.01 \mu \mathrm{Hz}$ following the four years of monitoring. It is thanks to this excellent frequency precision that the detection of rotational splitting in slow rotators became a reality (Pápics et al. 2014), with major progress and 
future potential in the seismic modelling of massive stars, as outlined below for two recently discovered pulsators.

Despite these observational studies for hundreds of OB dwarfs, only 16 of them could be modelled according to the scheme in Fig. 1 was achieved, the major obstacle being the lack of identification of the pulsation modes. However, several promising new cases based on four years of Kepler data are currently emerging. As a side remark, the detection of the numerous pulsation modes at low frequency in so many OB dwarfs, which was not possible from ground-based data, prompted the need for additional excitation mechanisms because the classical heat mechanism is not able to explain all the detected oscillations. One way to solve this is to increase the opacity, either globally in the star or in the excitation layer (Salmon et al. 2012; Walczak et al. 2013). A promising new excitation mechanism concerns stochastic gravity modes triggered by core convection (Belkacem et al. 2010; Samadi et al. 2010; Saio 2011; Shiode et al. 2013), although it remains unclear what their amplitudes are at the stellar surface.

\subsection{Evolved Massive Stars}

The case of asteroseismology of evolved OB stars is far more challenging, but accordingly more interesting, with large future potential. The challenge is not only due to the longer oscillation periods (which can reach up to several months) but also because the oscillations are influenced by various poorly understood physical processes which take place in the outer atmosphere and wind of such objects, affecting the boundary conditions that are of importance for the theoretical predictions needed to perform the scheme in Fig. 1.

Seismic data of evolved OB stars are scarce, even in the space age of asteroseismology. Also on the front of supergiant photometric data from space, MOST delivered the first interesting case with a $37 \mathrm{~d}$ light curve of the B2Ib/II star HD 163899. This revealed the simultaneous excitation of pressure and gravity modes with frequencies below $30 \mu \mathrm{Hz}$ and amplitudes of a few mmag (Saio et al. 2006). A MOST campaign of $28 \mathrm{~d}$ dedicated to the B8Ia supergiant Rigel was too short to uncover all the gravity modes found from six years of spectroscopic monitoring with frequencies as low as $0.2 \mu \mathrm{Hz}$ and up to $12 \mu \mathrm{Hz}$ (Moravveji et al. 2012). Of a completely different nature is the $137 \mathrm{~d}$ CoRoT light curve of the B6I star HD 50064, which, combined with spectroscopy, led to the conclusion that periodic mass-loss episodes due to a variable large-amplitude mode seem to occur (Aerts et al. 2010b). Finally, a 26 d CoRoT light curve of the B8Ib star HD 46769 led to the detection of low-amplitude rotational modulation with a frequency of $2.4 \mu \mathrm{Hz}$ and an amplitude of some $100 \mu$ mag rather than pulsations (Aerts et al. 2013).

The only evolved massive star observed by the Kepler mission is the bright eclipsing binary V380 Cyg, which required a customized mask (Tkachenko et al. 2012). Its Kepler light curve, along with extensive time-resolved spectroscopy led to the detection of rotational modulation along with low-amplitude stochastic variability at low frequency in the primary (Tkachenko et al. 2014). This variability is of similar nature than the patterns detected in the three hottest CoRoT O dwarfs (Blomme et al. 2011) and is compatible with theoretical predictions for stochastic gravity waves excited by the convective core.

The detailed theoretical interpretation of the periodic oscillations in supergiants remains to be done. While Godart et al. (2009) showed that mass loss and large overshooting reduce the extent of an otherwise occurring intermediate convective zone, preventing the excitation of gravity modes due to a heat mechanism acting in the metal opacity bump, Daszyńska-Daszkiewicz et al. (2013) do find such modes irrespective of an intermediate convection zone or not. Theoretical work on the improvement of stellar structure models of evolved massive stars will benefit from the seismic and spectroscopic data, following the scheme in Fig. 1. 

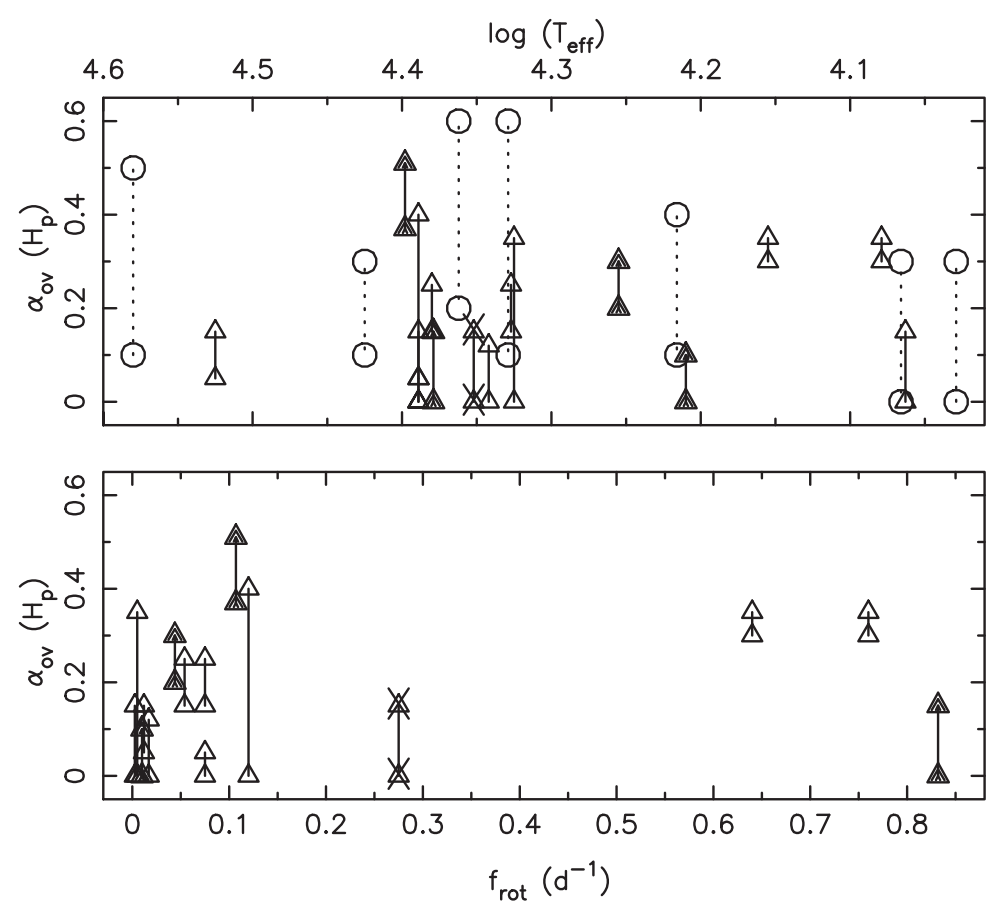

Figure 2. Core overshoot parameters from asteroseismology of OB stars as a function of the rotational frequency (lower panel) and effective temperature (upper panel), where single stars are indicated by open triangles and spectroscopic binaries by filled triangles; the magnetic pulsator is indicated with an additional cross. The estimates for the primaries of eclipsing binaries (Claret 2007) are indicated in the upper panel by circles connected with dotted lines.

\section{Seismic Modelling of OB Dwarfs}

Unlike solar-like stars and red giants, where ensemble asteroseismology can be achieved from scaling relations, the seismic modelling of massive stars requires a star-by-star treatment of the scheme in Fig. 1 and is thus immensely work-intensive. Aerts (2013) provided a compilation of the seismically derived stellar parameters according to Fig. 1, including the core overshoot value assuming the Schwarzschild criterion of convection and a fully mixed overshoot region, for 8 single and 3 binary non-emission OB dwarfs, among which one magnetic pulsator. The majority of these studies was based on multisite campaigns. In addition, seismic modelling was achieved for the late Be stars HD 181231 and HD 175869 (Neiner et al. 2012b). New studies since then were done for the $\beta$ Cep stars $\gamma$ Peg (Walczak et al. 2013) and $\sigma$ Sco (Tkachenko et al. 2014), as well as for the B8.3V star KIC 10526294 (Pápics et al. 2014). The latter star is a young slowly rotating $\left(P_{\text {rot }}=190 \mathrm{~d}\right)$ B star with 19 rotationally split and quasi-equally spaced dipole gravity modes detected in the 4-year Kepler light curve. Frequency inversion of its triplets leads to counter-rotation in its envelope (Triana et al., submitted). This star, along with the A-type pulsator KIC 11145123 whose envelope rotates slightly faster than its core, as revealed by rotationally split triplets and quintuplets (Kurtz et al. 2014), will undoubtedly give rise to future improvement of the input physics for stars of intermediate mass, because the current models cannot explain the observed pulsational properties.

A major finding from asteroseismology and of relevance for stellar evolution of massive stars is the need for extra mixing, enlarging the core masses of OB dwarfs, already from their early life. This was also hinted at long ago from modelling of the turn-off point 
of clusters, but this method has limited predictive power due to observational biases. Isochrone fitting of eclipsing binaries is a more powerful method to pinpoint $\alpha_{\text {ov }}$ (Claret 2007; Torres et al. 2014) independently from asteroseismology. In Fig. 2 we provide a compilation of the $\alpha_{\mathrm{ov}}$ values derived from asteroseismology for 16 OB dwarfs and from isochrone fitting for 7 unevolved eclipsing binaries. Only 9 of the 23 stars are compatible with the absence of core overshoot. Other than that, no obvious relation emerges. The same conclusion is found when comparing $\alpha_{\mathrm{ov}}$ with the mass.

\section{Spin-offs of Massive Star Asteroseismology}

\subsection{Macroturbulence}

The wings of the profiles of metal lines of a large fraction of OB stars cannot be explained by 1-dimensional atmosphere models, unless a macroturbulent velocity field is added (Símon-Díaz, these proceedings). Independently of whether one uses an isotropic or a radial-tangential Gaussian (Gray 2005) to describe this macroturbulence, supersonic speeds are often needed to bring the data in agreement with the model predictions, particularly for O stars and supergiants.

Aerts et al. (2009) suggested that the collective pulsational velocity broadening due to gravity modes could be a viable physical explanation for macroturbulence in massive stars. However, while B dwarfs are known to undergo pulsations with accompanying periodic line-profile variations (e.g., De Cat \& Aerts 2002; Aerts \& De Cat 2003), pulsations with the required frequencies and velocity amplitudes have yet to be firmly established for large samples of O stars and B supergiants. Indeed, the samples of OB stars observed by Simón-Díaz \& Herrero (2014) and Markova et al. (2014) reveal a diversity of timevariability in terms of line broadening, with the dwarfs having clear line-profile variations but the evolved stars not necessarily so while their line wings are strongly broadened. It therefore seems that convective velocities in the outer envelope are a more plausible explanation than pulsations for evolved stars (Símon-Díaz, these proceedings).

\subsection{Multivariate Statistical Analysis of Oscillations, Rotation, and Magnetic Field}

In the spirit of this symposium of considering an integrated approach, Aerts et al. (2014) made a multivariate study by combining spectropolarimetric, asteroseismic and spectroscopic observables derived from high-precision data for 64 galactic dwarfs. This 10dimensional data set is complete in $\log T_{\text {eff }}, \log g$, and $v \sin i$ and is more than $94 \%$ complete in the rotational frequency $f_{\text {rot }}$ and the magnetic field strength. An estimate of the nitrogen abundance is available for $59 \%$ of the targets while $32 \%$ of the stars have gravity-mode oscillation frequencies and $32 \%$ have pressure-mode oscillation frequencies. The stars without oscillations all have tight upper limits on the amplitudes.

One of the major aims of this study was to search for correlations between the nitrogen abundance and the other nine observables, from multivariate data analysis. This was achieved by applying a statistical technique called multiple imputation for the missing values, followed by linear regression from both backward and forward selection.

No significant correlation was found between the rotational observables $v \sin i$ or $f_{\text {rot }}$ and the nitrogen abundance. The latter did correlate with $\log T_{\text {eff }}$ and with the dominant acoustic oscillation frequency of the stars. Also a correlation between the dominant gravity-mode frequency and the magnetic field strength was found, but none of these two observables beared any relation with the nitrogen abundance.

While the sample may be prone to biases in the spectroscopic observables (cf. Przybilla, these proceedings), the study showed that rotation cannot be the only physical process to cause mixing in stellar interiors of galactic dwarfs and that magnetic fields are not 
a good alternative explanation. The role of other phenomena causing chemical mixing, such as oscillation modes or internal gravity waves (Mathis, these proceedings) could be a viable alternative or additional explanation.

\section{Conclusions and Future Prospects}

The past five years, photometric asteroseismic data improved in precision from mmag to $\mu \mathrm{mag}$ and in duty cycle from tens of percents to more than $90 \%$. Moreover, data became available for thousands of stars in almost all evolutionary stages, instead of being limited to a handful of solar-like stars, young B stars, subdwarfs and white dwarfs. As a consequence, asteroseismology opened a new window for stellar physics in such a way that stellar structure and evolution studies are currently observationally driven.

The discovery of gravity-mode oscillations in thousands of CoRoT and Kepler targets allows to probe the physics in and near stellar cores in both red giants (Eggenberger, these proceedings) and in massive stars. A major finding is that the internal stellar rotation, and along with it the angular momentum distribution inside stars, is not at all what current theories predict it should be. While the deviation between theory and observations in terms of the interior rotation profile in the A-type star KIC 11145123 and in the B-type star KIC 10526294 remains modest and can qualitatively be explained by the act of internal gravity waves (Mathis, these proceedings), the discrepancy for red giants is two orders of magnitude and requires at least one hitherto omitted strong coupling mechanism between the stellar core and the envelope (see also Maeder, these proceedings).

While asteroseismology of massive stars has been in action since more than a decade now, after the first seismic probing of the core overshooting (Aerts et al. 2003), the number of stars with detailed tuning of the internal physics is far too low and, moreover, restricted to dwarfs. This is first of all due to the limited number of such stars in the fields of CoRoT and Kepler (some tens compared to many thousands of giants), but also has to do with the lack of mode identification for the majority of OB-type stars observed in white-light space photometry. The problem of mode identification can only be overcome from extensive data sets of multicolour photometry or high-resolution high-precision spectroscopy of bright stars (Aerts et al. 2010b, Chapter 6), from the detection of period spacings of high-order gravity modes, (e.g., Degroote et al. 2010b; Pápics et al. 2012), from the occurrence of rotationally split multiplets (e.g., Aerts et al. 2003; Pamyatnykh et al. 2004; Briquet et al. 2007), or, ideally, a combination of the latter two cases (Pápics et al. 2014). The detection of rotationally split multiplets of high-order gravity modes was only achieved for two dwarfs with a convective core so far, and only after analysing four years of Kepler data (Kurtz et al. 2014; Pápics et al. 2014). These two stars will certainly be modelled in more detail in the coming months, with attention to improvement of the input physics of models, because the current theory cannot explain the details of the observed seismic behaviour (Moravveji, these proceedings).

A new opportunity in the coming months and years, are several hundreds of massive OB stars to be observed by the Kepler 2-wheel mission, a.k.a. K2 (Howell et al. 2014). It remains to be seen how many of those OB-type targets turn out to be pulsators with rotational splitting, given the rather limited time base of three months of continuous monitoring, but even a low percentage of the hundreds of submitted OB stars will open new avenues for massive star seismology including numerous evolved OB stars.

Another near-future avenue for improved seismic modelling is the addition of an accurate distance, and by implication a model-independent luminosity and radius estimate, from the ESA cornerstone mission Gaia (launched 19 December 2013 and currently in its 
commissioning phase). A direct accurate angular diameter measurement for bright stars from interferometry (van Belle, these proceedings), combined with an accurate parallax determination, would have similar capacity. The combination of asteroseismic data and a model-independent radius estimate implies a serious reduction in the uncertainties of the physical quantities of the stars from seismic inference, as outlined in detail by Cunha et al. (2007) and Huber et al. (2012) for stars with stochastically-excited oscillations. Given the current lack of accurate distances for massive stars, and of good calibrators for a high-precision interferometric radius determination of pulsating OB stars, progress in this area is to be expected in the next years.

Finally, the recently approved ESA M3 mission PLATO (Rauer et al. 2014), to be launched in 2024, will measure numerous OB-type stars in its very wide Field-of-View $\left(2250 \mathrm{deg}^{2}\right)$. This will include high-cadence $(2.5 \mathrm{~s})$ two-colour measurements of stars with visual brightness between 4 and 8 to be obtained by its two bright-star telescopes with a $95 \%$ duty cycle for a minimum of 2 years and, possibly, fainter OB stars in white light with a cadence of $32 \mathrm{~s}$ observable with all of the 32 normal telescopes, during the step-and-stare phase of the mission.

\section{References}

Aerts, C. 2013, in EAS Publications Series, Vol. 64, pp 323-330

Aerts, C., Christensen-Dalsgaard, J., \& Kurtz, D. W. 2010a, Asteroseismology, Astronomy and Astrophsyics Library, Springer Berlin Heidelberg

Aerts, C. \& De Cat, P. 2003, Space Sci. Revs 105, 453

Aerts, C., De Cat, P., Kuschnig, R., et al. 2006a, ApJ (Letters) 642, L165

Aerts, C., Lefever, K., Baglin, A., et al. 2010b, A\&A 513, L11

Aerts, C., Marchenko, S. V., Matthews, J. M., et al. 2006b, ApJ 642, 470

Aerts, C., Molenberghs, G., Kenward, M. G., \& Neiner, C. 2014, ApJ 781, 88

Aerts, C., Puls, J., Godart, M., \& Dupret, M.-A. 2009, AESA 508, 409

Aerts, C., Simón-Díaz, S., Catala, C., et al. 2013, A\&GA 557, A114

Aerts, C., Thoul, A., Daszyńska, J., et al. 2003, Science 300, 1926

Balona, L. A., Pigulski, A., Cat, P. D., et al. 2011, MNRAS 413, 2403

Beck, P. G., Bedding, T. R., Mosser, B., et al. 2011, Science 332, 205

Beck, P. G., Montalban, J., Kallinger, T., et al. 2012, Nature 481, 55

Bedding, T. R., Mosser, B., Huber, D., et al. 2011, Nature 471, 608

Belkacem, K., Dupret, M. A., \& Noels, A. 2010, A\&A 510, A6

Blomme, R., Mahy, L., Catala, C., et al. 2011, A\&A 533 , A4

Briquet, M., Aerts, C., Baglin, A., et al. 2011, A\&A 527, A112

Briquet, M., Morel, T., Thoul, A., et al. 2007, MNRAS 381, 1482

Cameron, C., Saio, H., Kuschnig, R., et al. 2008, ApJ 685, 489

Casagrande, L., Silva Aguirre, V., Stello, D., et al. 2014, ApJ 787, 110

Chaplin, W. J., Basu, S., Huber, D., et al. 2014, ApJS 210, 1

Chaplin, W. J., Sanchis-Ojeda, R., Campante, T. L., et al. 2013, ApJ 766, 101

Claret, A. 2007, A\&A 475, 1019

Corsaro, E., Stello, D., Huber, D., et al. 2012, ApJ 757, 190

Cunha, M. S., Aerts, C., Christensen-Dalsgaard, J., et al. 2007, A\&\&A Rev. 14, 217

Daszyńska-Daszkiewicz, J., Ostrowski, J., \& Pamyatnykh, A. A. 2013, MNRAS 432, 3153

De Cat, P. \& Aerts, C. 2002, A\&3A 393, 965

Debosscher, J., Blomme, J., Aerts, C., \& De Ridder, J. 2011, A\&A 529, A89

Debosscher, J., Sarro, L. M., López, M., et al. 2009, A\& $A$ 506, 519

Degroote, P., Acke, B., Samadi, R., et al. 2011, A\&A 536, A82

Degroote, P., Aerts, C., Baglin, A., et al. 2010a, Nature 464, 259

Degroote, P., Aerts, C., Ollivier, M., et al. 2009a, A\&\&A 506, 471 
Degroote, P., Briquet, M., Auvergne, M., et al. 2010b, A\&A 519, A38

Degroote, P., Briquet, M., Catala, C., et al. 2009b, A\&SA 506, 111

Deheuvels, S., Doğan, G., Goupil, M. J., et al. 2014, A\&A 564, A27

Desmet, M., Frémat, Y., Baudin, F., et al. 2010, MNRAS 401, 418

Diago, P. D., Gutiérrez-Soto, J., Auvergne, M., et al. 2009, A\&̋A 506, 125

Emilio, M., Andrade, L., Janot-Pacheco, E., et al. 2010, A\&A 522, A43

Gilliland, R. L., McCullough, P. R., Nelan, E. P., et al. 2011, ApJ 726, 2

Godart, M., Noels, A., Dupret, M.-A., \& Lebreton, Y. 2009, MNRAS 396, 1833

Gray, D. F. 2005, The Observation and Analysis of Stellar Photospheres, 3rd Edition, Cambridge University Press

Gruber, D., Saio, H., Kuschnig, R., et al. 2012, MNRAS 420, 291

Gutiérrez-Soto, J., Floquet, M., Samadi, R., et al. 2009, A\&A 506, 133

Handler, G., Matthews, J. M., Eaton, J. A., et al. 2009, ApJ (Letters) 698, L56

Hekker, S., Elsworth, Y., De Ridder, J., et al. 2011, A\&SA 525, A131

Howell, S. B., Sobeck, C., Haas, M., et al. 2014, PASP 126, 398

Huat, A.-L., Hubert, A.-M., Baudin, F., et al. 2009, A\&A 506, 95

Huber, D., Bedding, T. R., Stello, D., et al. 2011, ApJ 743, 143

Huber, D., Chaplin, W. J., Christensen-Dalsgaard, J., et al. 2013, ApJ 767, 127

Huber, D., Ireland, M. J., Bedding, T. R., et al. 2012, ApJ 760, 32

Kallinger, T., Mosser, B., Hekker, S., et al. 2010, A\&A 522, A1

Kurtz, D. W., Saio, H., Takata, M., et al. 2014, MNRAS, in press (arXiv1405.0155)

Lebreton, Y. \& Goupil, M.-J. 2014, A\&A , in press (arXiv1406.0652)

Mahy, L., Gosset, E., Baudin, F., et al. 2011, A\&A 525, A101

Markova, N., Puls, J., Simón-Díaz, S., et al. 2014, A\&A 562, A37

Miglio, A., Chiappini, C., Morel, T., et al. 2013, MNRAS 429, 423

Moravveji, E., Guinan, E. F., Shultz, M., Williamson, M. H., \& Moya, A. 2012, ApJ 747, 108

Mosser, B., Goupil, M. J., Belkacem, K., et al. 2012, Astronomy \& Astrophysics 548, A10

Neiner, C., Floquet, M., Samadi, R., et al. 2012a, A\&A 546, A47

Neiner, C., Gutiérrez-Soto, J., Baudin, F., et al. 2009, A\& A 506, 143

Neiner, C., Mathis, S., Saio, H., et al. 2012b, A\&A 539, A90

Pamyatnykh, A. A., Handler, G., \& Dziembowski, W. A. 2004, MNRAS 350, 1022

Pápics, P. I., Briquet, M., Auvergne, M., et al. 2011, A\&A 528, A123

Pápics, P. I., Briquet, M., Baglin, A., et al. 2012, A\&A 542, A55

Pápics, P. I., Moravveji, E., Aerts, C., et al. 2014, A\&A A in press (arXiv1407.2986)

Rauer, H., Catala, C., Aerts, C., et al. 2014, Experimental Astronomy, in press (arXiv1310.0696)

Saio, H. 2011, MNRAS 412, 1814

Saio, H., Cameron, C., Kuschnig, R., et al. 2007, ApJ 654, 544

Saio, H., Kuschnig, R., Gautschy, A., et al. 2006, ApJ 650, 1111

Salmon, S., Montalbán, J., Morel, T., et al. 2012, MNRAS 422, 3460

Samadi, R., Belkacem, K., Goupil, M. J., et al. 2010, ApESSS 328, 253

Sarro, L. M., Debosscher, J., Aerts, C., \& López, M. 2009, A $\& A$ 506, 535

Sarro, L. M., Debosscher, J., Neiner, C., et al. 2013, AESA 550, A120

Shiode, J. H., Quataert, E., Cantiello, M., \& Bildsten, L. 2013, MNRAS 430, 1736

Simón-Díaz, S. \& Herrero, A. 2014, A\& A 562, A135

Stello, D., Huber, D., Bedding, T. R., et al. 2013, ApJ (Letters) 765, L41

Thoul, A., Degroote, P., Catala, C., et al. 2013, A\& A 551, A12

Tkachenko, A., Aerts, C., Pavlovski, K., et al. 2014, MNRAS 442, 616

Tkachenko, A., Aerts, C., Pavlovski, K., et al. 2012, MNRAS 424, L21

Torres, G., Vaz, L. P. R., Sandberg Lacy, C. H., \& Claret, A. 2014, AJ 147, 36

Van Eylen, V., Lund, M. N., Silva Aguirre, V., et al. 2014, ApJ 782, 14

Walczak, P., Daszyńska-Daszkiewicz, J., Pamyatnykh, A. A., \& Zdravkov, T. 2013, MNRAS 432, 822 


\section{Discussion}

Chieffi: Can you provide the mass of the convective core of the stars when you do seismic modelling?

AERTS: Sure, since we so far considered the Schwarzschild criterion and added a fully mixed overshoot region, we could have taken the full core mass as a quantity, rather than the overshoot parameter. We will take it up when we redo a homogeneous remodelling of all 16 stars mentioned in Sect. 3, because these results have been assembled over the past decade and not all the studies contain this information. Also, the modelling has been done with improved input physics over the years.

MEYNET: In massive stars, the convective core decreases in mass as a function of time, so to provide a constraint to the stellar models, you need to determine the size of the core, as well as the age and initial mass of the star.

AERTS: Correct. That is precisely what we do in seismic modelling (cf. Aerts 2013): we start from a huge grid of stellar models computed from the Hayashi track up the H-shell burning, with the following parameters: $M$, initial $X$ and $Z$, age, and core overshoot parameter. The grid is so dense that the pulsation frequencies from one model to the next on each evolutionary track, differs less than the precision of the detected oscillation frequencies. In this huge grid, we select the models that best fit the seismic data according to Fig. 1. In that way, we get high-precision estimates of the four parameters, for the adopted input physics. Point taken that the theoreticians want us to provide not only the mass and the age, but also the size of the core and we shall document it in future papers (cf. point by Alessandro Chieffi).

SAIO: Is there any correlation between the core overshooting parameter and the rotational frequency of the star?

AERTS: Cf. Fig. 2 in the paper: not really. The diversity of this parameter for massive stars is much larger than anticipated before, which points out that the extra mixing necessary to explain the seismic data is probably caused by various mixing phenomena whose efficiency differs from star to star. This is in line with the few results available from isochrone fitting of massive eclipsing binaries, where one relies on the input physics in the same way as for seismic modelling. These have now been added in the plot, for comparison with the seismic estimates.

NoELS: When using an inversion procedure for the SPB KIC 10526294, you already need to have a good estimate of the density distribution. With a counter-rotating envelope, the density is likely different so it seems to me that an iteration process should be made in order to achieve the "true" rotation profile.

AERTS: Completely agreed that our result cannot be but a 1st step and we now need to plug in the profile to see what kind of effect it would have on the stellar structure, in particular on the mixing and on the angular momentum distribution it will imply. For the moment, we did not yet do such an iteration. Where we are today, is that we found stellar models that explain the 19 equally spaced zonal modes satisfactorily, all the best models have kernels with a strongly differing probing power in the stellar envelope, and the inversion gives consistent results independently of which of the better models we take to compute the kernels. Moreover, we took a broad range in the smoothing parameter 
that occurs in the inversion procedure to make sure the result is independent of the choice. In addition, we did forward modelling based on the computed $\Omega(r)$ to check if the observed splittings, including their asymmetries, are reconstructed and that is indeed the case. We will move on to the next steps in this subject, keeping in mind that Kepler also delivered other types of main-sequence pulsators which do not lead to a rotation profile as expected from theory. As far as I am aware, only internal gravity waves can explain the observations of the newly discovered SPB KIC 10526294.

DE KoTER: The Hunter diagram shows that there are groups of stars that we do not understand at all, e.g., slowly rotating N-enriched objects. I am surprised that your statistical study shows that there is a correlation between $\mathrm{N}$ and $T_{\text {eff }}$. Your stars cover the entire main sequence and therefore age enters because age and $\mathrm{N}$ are coupled through rotational mixing. Stars in the Hunter diagram tend to be near-TAMS stars.

AERTS: Our sample indeed covers the entire main sequence (cf. plot in our paper). The sample selection was purely based on the availability of high-precision observables and our explicit aim was not to rely on any theoretical predictions. Note that Rivero González et al. (2012, A\&A, 537, A79) came to the same conclusion from a completely independent study of LMC O stars, for which they found that mixing occurs already early on in the core-hydrogen burning phase for a considerable fraction of $\mathrm{O}$ stars and hence cannot be due to rotational mixing alone.

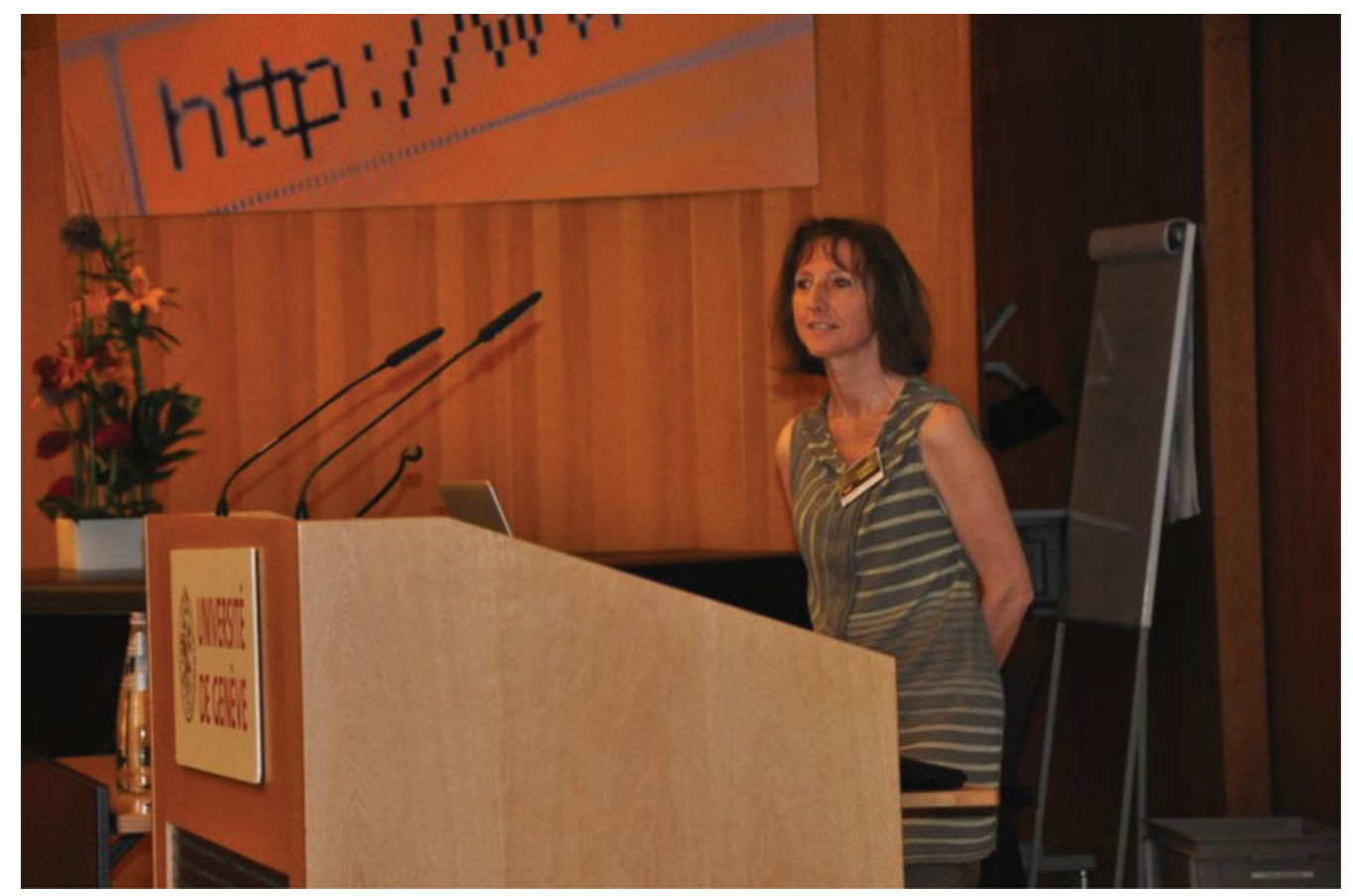

Conny Aerts 